from the US National Science Foundation to Yale University Observatory.

Phillip K. Lü

JAMES H. HunTER, jun.

Yale University Observatory,

New Haven, Connecticut.

Reccived January 13, 1969.

${ }^{1}$ Sandage, A. R., Astrophys. J., 144, $123+$ (1966).

${ }^{2}$ Angione, 1. J., Publ. Astro. Soc. Pacific, 80, 339 (1968).

\section{Direct Current Pre-breakdown Phenomena in Liquid Nitrogen}

WE have used divergent field (wire-cylinder and pointplan€) electrode configurations to study the emission under high field of electrons from a metal into a liquid dielectric -in particular, liquid nitrogen. After outgassing the emitting electrode under high vacuum, high purity gas is condensed under slight overpressure, while taking proper steps to avoid the presence of dust or impurities.

Such a system enables a very small fluid volume to withstand enormous electric fields with a moderate applied voltage. Furthermore, the stressed volume is so small that the probability that cosmic rays will induce ionization in this volume is negligible.

Preliminary work with point-plane geometry ${ }^{1}$ showed that emission does not start-even if the stress at the high field electrode is higher than the usually accepted break.

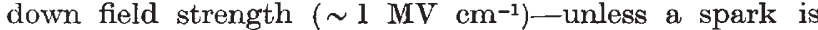
triggered, or contamination is introduced, in the cell.

In the work described here the measuring cell we used consists essentially of two electrodes (a point in front of a plane) in a demountable 'Pyrex' envelope. The tip, prepared as a field emitter, can be removed. It can be heated in situ by Joule effect and then observed by electron microscopy, a procedure incompatible with the usual technique of field emission microscopy. The counterclectrode consisted of a platinum foil or a platinum grid.

After evacuation, the cell was dipped in liquid nitrogen to improve the vacuum-the tip was heated, the $I(V)$ characteristics in vacuo were measured, the liquid was introduced and the $I(V)$ characteristics were measured with the filled cell.

The emission into pure liquid nitrogen triggered itself rather abruptly, at a voltage 2.5 to 4 times higher than the threshold for emission in vacuo. Before this occurred no current was detectable in the liquid, even for applied voltages corresponding to a local field in front of the tip

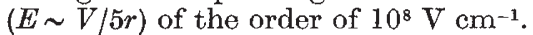

Once triggered, the current in liquid nitrogen varied linearly with the square of the applied voltage, and roughly with the reciprocal point-plane distance. It depended relatively little on the metal used for the point. Substituting a grid for the flat plane counter electrode increased the current by about 25 per cent.

Sustained emission considerably eroded the tip, the radius of which could increase from less than $300 \AA$ to $1 \mu \mathrm{m}$. If the applied voltage was lowered during emission, the current cut off at a voltage $V_{D}$. Then, if the voltage was raised, the current reappeared at a voltage close to $V_{D}$. If the liquid was removed without disturbing the point, however, a voltage of about $2 V_{D}$ was required to resume the flow of current.

When the current reached about $10^{-7} \mathrm{~A}$, a faint greenish light could be seen in front of the tip with the naked eye. Furthermore, careful observation of small bubbles suggested that there was a liquid motion, which was detected in another cell by rotating a small propeller. These effects were probably present, though not detectable, as soon as the current is initiated.
Current emission into liquid nitrogen also occurred in the opposite polarity making the tip positive, and it behaved similarly. Current initiation, however, occurred in rather different conditions.

The part played by field emission in our experiments is hard to assess. The potential barrier seemed unexpectedly opaque in the presence of liquid nitrogen, and one might speculate that initiation occurs when potential barriersat the cathode or in the molecules-are lowered by the field to electronic energy levels. Accurate experiments on the influence of the polarity are necessary to check the validity of these speculations.

Once current emission has been initiated, the electrical power dissipated into the liquid is higher than $10^{-5} \mathrm{~W}$. Such a power is sufficient, in principle, to vaporize $4 \times 10^{-8} \mathrm{~cm}^{3}$ of liquid nitrogen each second at $77^{\circ} \mathrm{K}$ under atmospheric pressure, but the electrostrictive overpressure reduces vaporization, and part of the injected power is converted into work against viscous forces.

As the linear variation of $\sqrt{ } I$ against the applied voltage shows, the current is space-charge-limited ${ }^{2}$ and the carrier mobility is practically independent of the field. From the slope of the $\sqrt{ } I$ against $V$ lines, and the theory of spacecharge-limited current in spherical configuration taking into account the non-spherical shape of the tip, values for the mobility between 1 and $2 \times 10^{-3} \mathrm{~cm}^{2} \mathrm{~V}^{-1} \mathrm{~s}^{-1}$ closely distributed around 1 and $1 \cdot 4 \times 10^{-3} \mathrm{~cm}^{2} \mathrm{~V}^{-1} \mathrm{~s}^{-1}$, have been obtained. Similar treatment of the data of ref. 1 yields consistently similar values: $(1 \cdot 2 \pm 0 \cdot 2) \times 10^{-3} \mathrm{~cm}^{2} \mathrm{~V}^{-1} \mathrm{~s}^{-1}$.

The liquid is dragged along the direction of the point axis in the point-plane configuration, and radially in the wire-cylinder configuration, so the agreement between the results suggests that liquid drag plays a small part.

Some of our results are in qualitative agreement with findings obtained with deliberately contaminated nitrogen ${ }^{3}$.

The important, unexpected result of this work is that the local field in front of the tip may reach $100 \mathrm{MV} \mathrm{cm}^{-1}$ (the hypothesis of field reduction by space charge is discarded by the low current level before any pre-breakdown phenomenon becomes detectable). This figure seems less extravagant if it is remembered that the "breakdown field" varies inversely with the interelectrode distance ${ }^{4-7}$. In our configuration, we actually work with "effective" gaps (distances in which the liquid is highly stressed) 100 times smaller than the smallest gaps used in parallel plane, uniform field configurations.

This supports and confirms an idea already discussed ${ }^{8}$ according to which the "breakdown field" has no intrinsic physical meaning. Only the field able to induce ionization of the molecules (135 MV $\mathrm{cm}^{-1}$ for nitrogen ${ }^{9,10}$, a value probably reached in our experiments) has a physical mean-

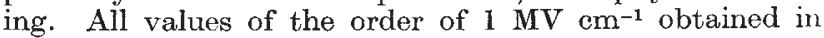
uniform field result from artefacts such as the presence of ions produced by cosmic radiations, or of fibres, bubbles and so on.

This work was supported in part by the Délégation Générale à la Recherche Scientifique et Technique.

\section{R. Coelho}

P. Sibillot

Laboratoire de Génie Electrique,

Associé an CNRS,

Fontenay-aux-Roses, France.

Received November 4, 1968.

${ }^{1}$ Coelho, R., CR Acad. Sci., 262 B, 992 (1966).

'Halpern, B., and Gomer, G., J. Chem. Phys, 43, 1069 (1965).

8 Byatt, S. W., and Sccker, P. E., Brit. J. App. Phys. II, 1, 1011 (1968).

${ }^{4}$ Sharbaugh, A. H., et al., J. App. Phys., 25, 1480 (1954); 26, 434 (1955).

${ }^{5}$ Goldschvartz, J. M., and Blaisse, B. S., B rit. J. App. Phys., 17, 1083 (1966)

${ }^{6}$ Kuchinskii, G. S., Sov. Phys. Techn. Phys., 11, 964 (1967).

7 Gallagher, T. J., Coll. Intern. CNRS, 179 (Grenoble, 1968).

${ }^{8}$ Brigneli, J. E., Proc. Inst. Elect. Eng., 113, 1683 (1966).

${ }^{\circ}$ Muller, E., Field Ionization and Field Ion Microscopy Advances in Electronics and Electron Physics, 83 et seq. (Academic Press, 1960). 10 Henson, B. L., Phys. Rev.,135, A 1002 (1964). 